\title{
A Potential and Proficient Relay Node Selection (PPRS) Mechanism for MANET Performance Enhancement
}

\author{
N. Snehalatha ${ }^{1 *}$ and Paul Rodrigues ${ }^{2}$ \\ 'School of Computing, SRM University, Chennai - 603203, Tamil Nadu, India; \\ snehalatha.n@ktr.srmuniv.ac.in \\ 2Department of CSE, College of CS, KKU, Abha, Saudi Arabia
}

\begin{abstract}
Objectives: The main objective is to propose a Potential and Proficient Relay Node Selection (PPRS) Mechanism for progressing MANET Performance. Method/Analysis: Although, Routing issues are under constant study for many years now already. The literature shows that the issue of performance lacking in highly dynamic MANETs has not been completely eliminated. Consequently, the main method adapted by PPRS mechanism is the individual next hop selection consecutively along with the estimation of success probability for selecting that node. Findings: The consequent and persistent next hop selection builds up MANET routes while assuring great communication statistics even under highly dynamic conditions. Novelty/Improvement: The performance of the PPRS mechanism is contrasted with existing EDEAR mechanism using simulations in the network simulator.
\end{abstract}

Keywords: Energy, MANET, Mobility, Relay Node, Throughput

\section{Introduction}

With the advancement found in MANET technology into almost all fields of existence including science, commerce, domestic and educational realms, the research in MANET. Loo et al, 2011 have also expanded its bounds to a great extent $\frac{1}{}$. As already known, a wireless ad hoc network is merely a collection of devices without wired connectivity and do not require any centralized managing device to perform as a communicating technology ${ }^{2}$.

The network topology keeps changing dynamically in a MANET and so does the configuration of the links change dynamically as well. The only means for them to seamlessly communicate in spite of the changing links is to be able to design a protocol that can deal with the rapid changes in an optimal strategy ${ }^{3}$.

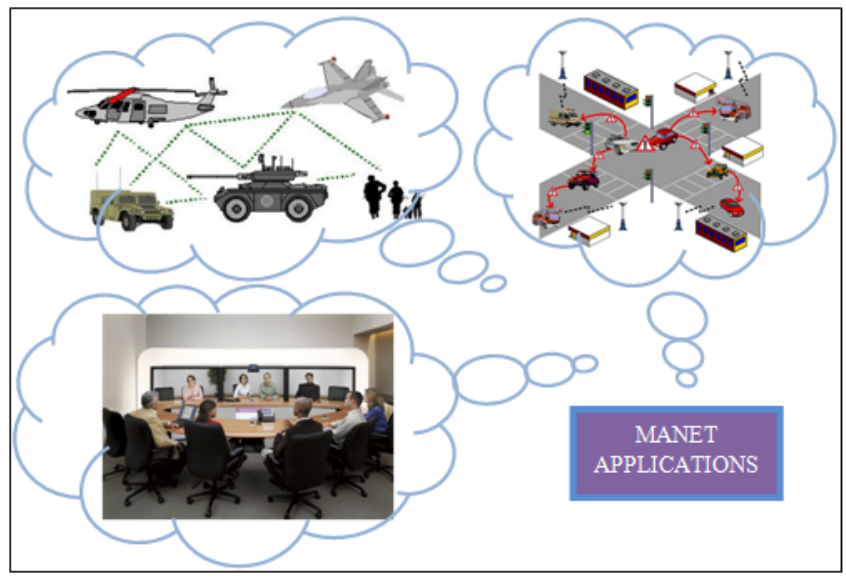

Figure 1. General applications of a MANET 
The first and foremost attribute to implement this method is to design a strategy to find the presence of the other devices within the communication range of the searching node. The different requirements of the searching node, the purposes for which the ad hoc network is formed like service access, data sharing and software application exchanges, need to be evaluated and thereby processed within the minimum possible amount of time $e^{4}$. However, changes are unpredictable time and again. Therefore, it is a good approach to identify and correct any issues before any link breakage or unpredictable change can occur in MANETs 5 .

There are two ways of communication present in the MANET scenarios depending on the application and situation of the nodes. Unicasting can be observed in simple one to one scenarios like connecting to a wireless printer from a phone while in motion. Whereas, multicasting can be found in scenarios like military monitoring and emergency operations in addition to simple personal tasks if required as well.

Protocol Independent Multicast (PIM) $\underline{6}$ and Core Based Trees $(\mathrm{CBT})^{\underline{Z}}$ uses the notion of group-shared trees. The core-based group-shared tree protocol is used to distribute the packets from all the sources because it is a shortest-path tree rooted at some core node. The core node is also referred to as a centre node or a meeting point. Core nodes may be chosen from some preselected set of nodes or some heuristics may be employed to select core nodes. All these methods may appear facile but the complexity is increased to a substantial level.

In MANET Proactive Source Routing Protocol (PSR) is a very efficient routing protocol analyzed using network simulator ${ }^{-}$. This protocol is lightweight and proactive with increased source routing capability. The overhead present in this protocol is much reduces when compared to a large number of routing protocols based on the DV routing. The transportation of data across the nodes is very good as well. There are majorly three updates proposed in this technique: Route Update; neighbourhood trimming and streamlined differential update. When two nodes $u$ and $v$ are connected by edge $e=(u, v) \in E$ if they are close to each other and can directly communicate with given reliability. Given node $v, N(v)$ denotes its open neighborhood, i.e., $\{u \in V \mid(u, v) \in E\}$. Similarly, $N[v]$ to denotes its closed neighborhood, i.e., $N(v) \cup\{v\}$. The proactive update is distributed within all the nodes and is performed iteratively. The given update of the Breadth First Spanning Tree (BFST) happens several times within a single update interval so that a node can integrate new route information to its knowledge base faster. After a finite number of iterations of operation, each node in the network has constructed a BFST of the entire network rooted at itself since nodes are timer driven and synchronized as well. The fundamental approach is to send the entire update messages rarely than small messages enclosing the difference between the current and previous facts of a node's routing module. The performance of PSR proved to be better than protocols like OLSR (Optimized Link State Protocol) $)^{9}$, ExOR (Extremely Opportunistic Routing $)^{10}$, Destination Sequenced Distance Vector routing ${ }^{11}$ and Dynamic Source Routing ${ }^{12}$ protocols.

\section{Design of the Potential and Proficient Relay Node Selection (PPRS) Mechanism}

The design of the proposed method is achieved by the step by step evaluation of the various attributes of a node and then utilizing the same to build a consistent path to the destination. For the same reason, we first define various terms for individual node evaluation and then a separate strategy for utilization of the individual information of a node.

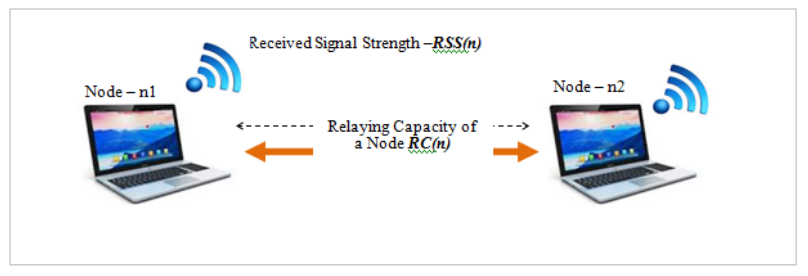

Figure 2. Estimating metrics for PPRS.

We propose to estimate the dynamic metrics to judge a node's individual ability to co-operate in a MANET communications. We analyze four major attributes that determine the success of a data transmission via the next hop of a neighbour.

A nested check list is created before the selection of every node. If these minimum criteria are passed, then the node will be allowed to be a next node for the current communication. Otherwise the protocol suggests taking a second possibility and so on until it is able to clear the minimum criteria. 


\subsection{Received Signal Strength Indicator (RSSI)}

The total power present in a received signal from a wireless device is said to be received signal strength. The Received Signal Strength indicator is generally measured between 0 to 100 for an IEEE 802.11 system using various tools like wire shark, insider, etc. However, the number is made available in the network simulator tool for research purposes. Therefore, this is the major criterion or the first hurdle for a node to clear before it could be considered for routing. It is dynamically estimated by the previous hop of every node as $R S S I(n)$, where $0 \leq R S S I(n) \leq 100$ for all nodes $\mathrm{n} \times \mathrm{N}$.

\subsection{Processing Node Capacity (C)}

The processing capacity of a node is estimated using the packet inflow and outflow rates in the proposed technique. The formula to estimate capacity of a node is derived from the fact that the difference between the number of packets sent and received is proportional to the capability of a node. The node capacity therefore can be given as (1),

$$
C(n) \alpha\left(\operatorname{Recv}_{P K T}-\operatorname{Sent}_{P K T}\right)
$$

Similarly, the degree of the node $k$ is also proportional to the node capacity as given in the expression (2)

$$
C(n) \alpha k
$$

Generally, the capacity of a node is assessed at regular intervals of time $t$. The time at which packets were received by a node $\left(T_{R E C V}\right)$ and time at which it processes its outgoing packets into the radios $\left(T_{\text {SENT }}\right)$. Therefore, the total processing time taken by a node which includes both the incoming and outgoing packets, i.e. the time duration taken by the packet to in transit in a node can be obtained by the difference between $T_{R E C V}$ and $T_{S E N T}$. The processing capacity of a node $\mathrm{C}$ is inversely proportional to this time difference as illustrated in the expression (3).

$$
C(n) \propto \frac{1}{T_{S E N T}-T_{R E C V}}
$$

Hence we combine the expressions (1), (2) and (3) to obtain an equation (4), an estimate for $\mathrm{C}(\mathrm{n})$,

$$
C(n)=P_{K} \frac{\left(\operatorname{Rec}_{P K T}-\operatorname{Sent}_{P K T}\right) \times k}{\left(T_{S E N T}-T_{R E C V}\right)}
$$

\subsection{Potential Link Duration}

This metric is to merely check if the next possible is so highly mobile that it is not suitable for picking up the information from the current hop. Therefore, relative velocity is used as a metric to filter out the highly mobile nodes. Potential Link Duration is the expected time the node has link with the current node. This is measured using the formula in equation (5).

$$
P L D(n)=R S S I \times\left(R S_{\text {CurrentHop }} \pm R S_{\text {NextHop }}\right)
$$

Here, $R S_{\text {CurrentHop }}$ and $R S_{\text {NextHop }}$ are the relative speeds of the current and the next hops respectively, while establishing a route in MANETs. If the direction of motion of the current hop and the next hop are towards each other, then there is addition in their speeds. If they are moving against each other, then the difference of their relative speed is obtained in the equation (4). Therefore we define $P L D(n)$ as a product of the RSSI and the Relative speeds.

\subsection{Probability of Success $P_{S}$}

The probability of success $P_{s}(n)$, is the contribution the individual node can make to the success of the entire communication. The probability of Success is taken between 0 and 1 for the PPRS mechanism. This is the final metric that decides whether this node causes success in the transmission of data or not.

\section{Working Strategy of PPRS}

The working strategy of PPRS is novel in terms of presenting a multi conditional assessment for each node selection. The consecutive check in the metrics is performed in order to pass a node as the relay node for next hop communication.

The above steps show how the consecutive check of a node allows in the efficient next hop selection with respect to the entire network communication. Using this method, it is possible to filter out inefficient nodes and thereby improve MANET communication. 


\section{Steps involved in the PPRS Method:}

Step 1: Create a Network Scenario in NS2

Step 2: Configure nodes as Mobile nodes according to IEEE 802.11 Standard

Step 3: Model four metrics for the assessment of nodes:

Received Signal Strength Indicator

Potential Link Duration, $P L D(n)$

Processing Capacity of a Node

Probability of Success, $P_{S}(\boldsymbol{n})$

Step 4: Get consecutive neighbour sets from source if it wants to send information to destination

Step 5: Sort the nodes list according to the distance

Step 6: Get the shortest distance node and check for the RSSI first

Step 7: If $\operatorname{RSSI}(n)>R S S I_{\text {Threshold }}$,

then check $C(n)$, If $C(n)>C_{\text {Threshold }}$

then check $P L D(n)$, If $P L D(n)>P L D_{\text {Threshold }}$

Select $\mathbf{n}$ as next hop and transmit data

$$
\text { then check } P_{S}(n) \text {, If } P_{S^{\prime}}(n)>P_{S^{-} \text {Threshold }}
$$

; Else Goto Step 8

; Else Goto Step 8

$$
\text { Else Goto Step } 8
$$

Step 8: Else pick another node and Repeat step 6.

Step 9: If destination is reached, end data transmission

Step 10: If no more data to transmit, Exit

\section{Simulation Analysis}

The simulation of the EDEAR and PPRS schemes are performed in the Network Simulator tool to analyze the network performance and energy efficiency. NS2 is a discrete event simulator in which a scenario is created to perform simulations of the proposed enhancement over the comparison baseline of existing. The simulation parameters are listed in the Table 1. To analyze the network performance, the packet delivery ratio, loss ratio and delay are measured.

Table 1. Simulation parameters of PPRS and EDEAR

\begin{tabular}{|l|c|}
\hline Parameter & Value \\
\hline Simulation Time & $100 \mathrm{~ms}$ \\
\hline Number of nodes & 30 \\
\hline Routing scheme & PPRS and EDEAR \\
\hline Traffic model & CBR \\
\hline Initial Energy & $1 \mathrm{~J}$ \\
\hline Simulation Area & $500 \times 500$ \\
\hline Transmission range & $200 \mathrm{~m}$ \\
\hline
\end{tabular}

\subsection{Packet Delivery Ratio}

The Packet Delivery Ratio (PDR) indicates how efficiently data is transmitted across the network. It is the ratio of the total packets received to those sent. It can otherwise be called as the delivery success ratio and is estimated by the equation (6) that follows.

$$
P D R=\frac{\text { SPackets Recv }}{\text { Packets Sent }}
$$

The packet delivery of the PPRS is greater than that of the EDEAR protocol. This is because the reliability increases when the efficient nodes are selected for data transmission using the potential in the node.

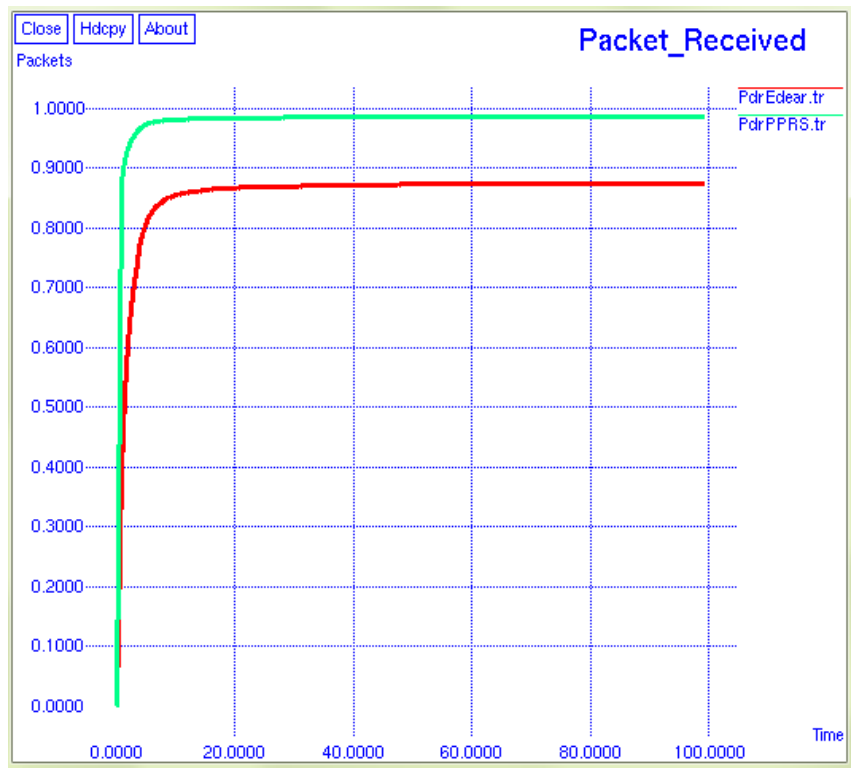

Figure 3. Packet delivery of EDEAR and PPRS.

\subsection{Packet Loss Ratio}

The total loss occurring in the network is determined by the Packet Loss Ratio (PLR). It is the ratio of the total packets lost to the total number of packets sent and can be calculated by the formula in equation (7).

$$
P L R=\frac{\text { SPackets Loss }}{\text { Packets Sent }}
$$




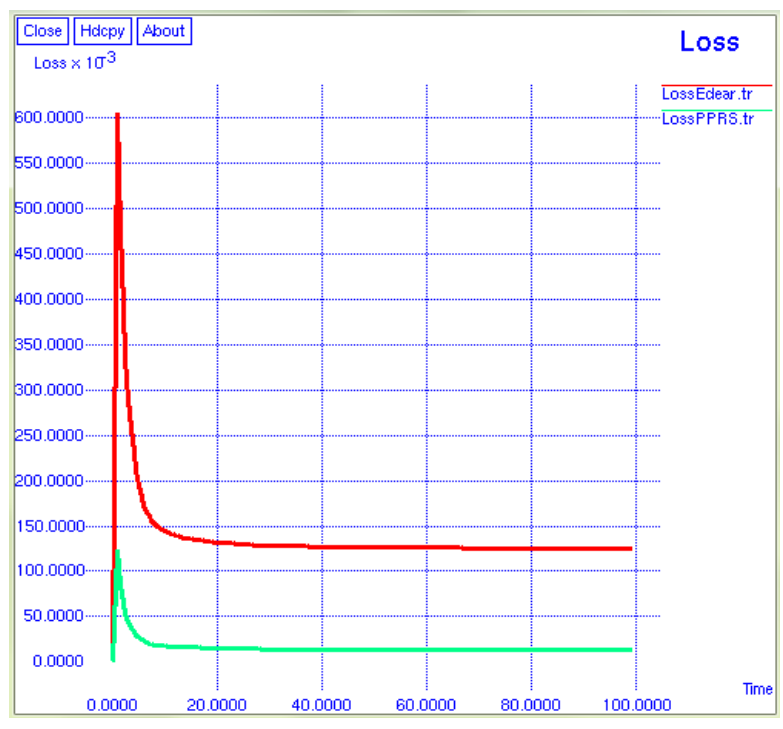

Figure 4. Packet loss ratio of EDEAR and PPRS.

The packet loss occurred in the PPRS is slightly reduced due to the reliability increase after potential node check similar to that of the packet delivery.

\subsection{Throughput}

Throughput is defined as the successful transmission of total number of packets in bytes/bits across the network. The throughput for the EDEAR and PPRS mechanisms per node is shown in the Figure 5. It can be observed from the Figure 5 that the throughput of the PPRS mechanism is greater than that of the EDEAR mechanism. Therefore it is possible to improve the number of successful transmission by eliminating the inefficient nodes while routing.

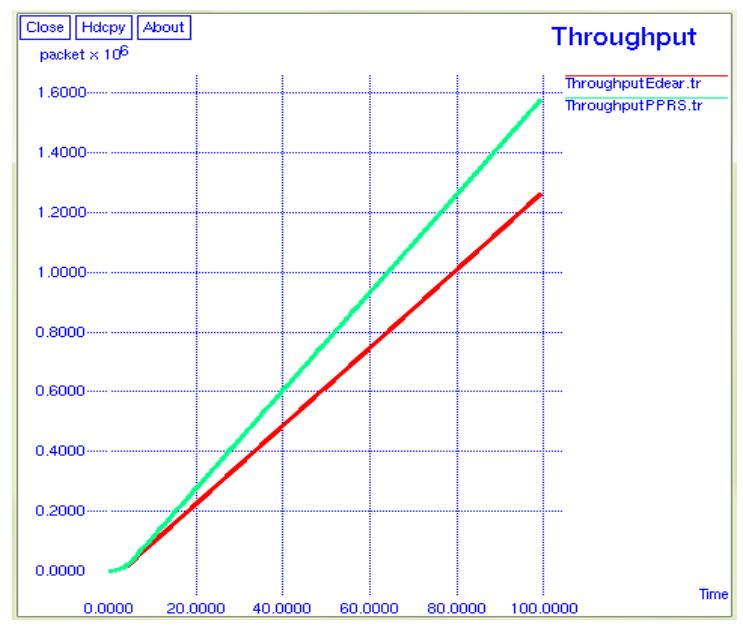

Figure 5. Throughput of EDEAR and PPRS.

\subsection{Delay}

The total delay taken by every node for processing the packets and sending to the next hop is taken as the delay in the network here. Therefore, the delay for the EDEAR and the PPRS mechanisms are shown in the Figure 6 which describes the delay of the PPRS mechanism is greater than that of the EDEAR protocol. This difference is due to the number of parameters measured in EDEAR is comparatively higher than the metrics measured in the PPRS mechanism.

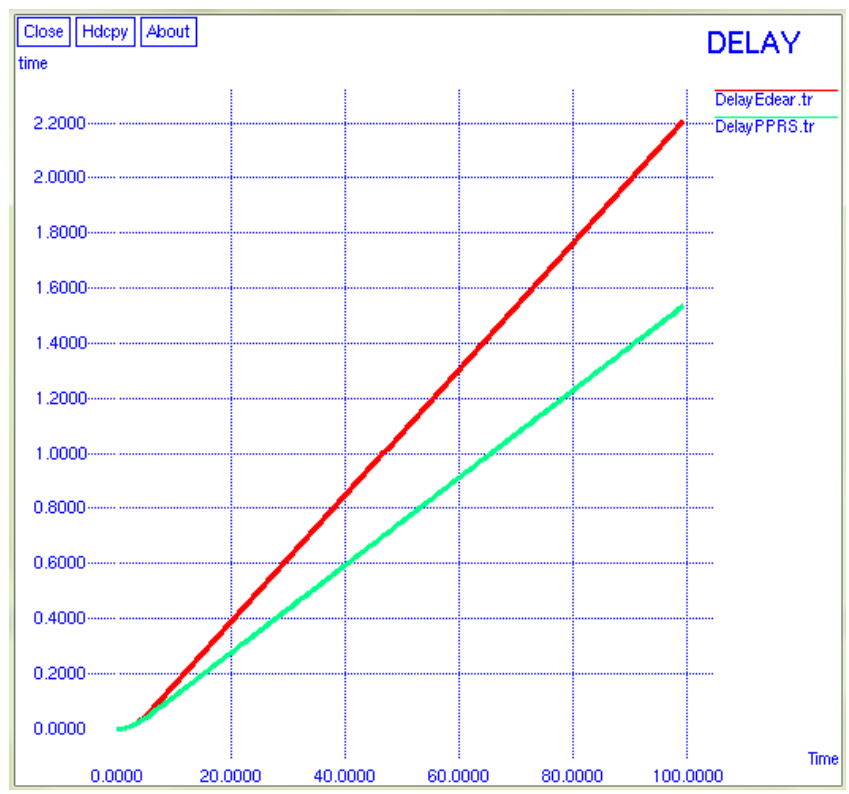

Figure 6. Delay of EDEAR and PPRS.

\section{Conclusion}

This paper proposes a Potential and Proficient Relay Node Selection (PPRS) Mechanism for progressing MANET Performance. The main focus of this mechanism lies in the individual next hop selection along with the estimation success probability for selecting that node. The method employs four metrics: Received Signal Strength Indicator, Processing Capacity of a Node, Potential Link Duration, Probability of Success, to ensure the efficient next hop selection in MANET. This method has proven its performance over the EDEAR protocol and therefore suits the mobile nature of MANETs to a good extent. Future works include the investigation of the other metrics $\frac{13,14}{14}$ for efficient communication in highly dynamic MANETs. 


\section{References}

1. Loo J, Mauri JL, Ortiz JH. Mobile ad hoc networks: Current status and future trends. CRC Press; 2011.

2. Haas ZJ, Deng J, Liang B, Papadimitratos P, Sajama S. Wireless ad hoc networks. Encyclopedia of Telecommunications; 2002.

3. Chlamtac I, Conti M, Liu JJN. Mobile ad hoc networking: Imperatives and challenges. Ad hoc Networks. 2003; 1(1):13-64.

4. Goyal P, Parmar V, Rishi R. Manet: Vulnerabilities, challenges, attacks, application. International Journal of Computational Engineering and Management. 2011; 11(2011):32-7.

5. Satyanarayana D, Rao SV. Link failure prediction QoS routing protocol for MANET. IET-UK International Conference on Information and Communication Technology in Electrical Sciences (ICTES 2007); 2007 Dec. p. 1031-6).

6. Adams A, Nicholas J, Siadak W. Protocol independent multicast-dense mode (PIM-DM): Protocol specification (revised) (No. RFC 3973); 2004.

7. Ballardie T, Francis P, Crowcroft J. Core Based Trees (CBT). ACM SIGCOMM Computer Communication Review. 1993 Oct; 23(4):85-95.
8. Wang Z, Chen Y, Li C. PSR: A lightweight proactive source routing protocol for mobile ad hoc networks. IEEE Transactions on Vehicular Technology. 2014; 63(2):859-68.

9. Clausen T, Jacquet P, Adjih C, Laouiti A, Minet P, Muhlethaler P, Viennot L. Optimized Link State Routing protocol (OLSR); 2003.

10. Biswas S, Morris R. Opportunistic routing in multihop wireless networks. ACM SIGCOMM Computer Communication Review. 2004; 34(1):69-74.

11. Perkins CE, Bhagwat P. Highly dynamic DestinationSequenced Distance-Vector routing (DSDV) for mobile computers. ACM SIGCOMM Computer Communication Review. 1994 Oct; 24(4):234-44.

12. Johnson, DB, Maltz DA. Dynamic source routing in ad hoc wireless networks. Mobile Computing. Springer US; 1996. p. 153-81.

13. Kumari DS, Sikamani KT. Communication based Clustering to detect selfish nodes in MANET. Indian Journal of Science and Technology. 2015; 8(20).

14. Akhtar MAK, Sahoo G. Behavior based high performance protocol for MANET. Indian Journal of Science and Technology. 2013; 6(10):5342-50. 